\title{
Multiple-Symbol Differential Sphere Detection for the Amplify-and-Forward Cooperative Uplink
}

\author{
Li Wang and Lajos Hanzo \\ School of ECS, University of Southampton, SO17 1BJ, UK. \\ Tel: +44-23-8059 3125, Fax: +44-23-8059 4508 \\ Email: $\{1 w 05 r, 1 h\} @ e c s . s o t o n . a c . u k ;$ http://www-mobile.ecs.soton.ac.uk
}

\begin{abstract}
It is widely recognized that the differential amplify-andforward (DAF) transmission scheme is capable of providing a superior performance compared to classic direct transmissions employing differential detection in slow-fading channels and in fact it may even outperform coherent detection aided systems relying on realistic imperfect channel estimates. However, in reality the channels connecting the multiple nodes of a cooperative system typically become time-selective due to the relative mobility of the cooperating terminals. Hence, the performance gain achieved may erode as the environment becomes more time-selective. On the other hand, multiple-symbol based differential sphere detection (MSDSD) has been proposed to mitigate the channelinduced performance loss suffered by classic direct transmission schemes employing the conventional differential detection (CDD) scheme. Hence in this paper, we specifically design the MSDSD based on a multidimensional tree search, which is capable of achieving a significant performance gain in the context of a DAF-aided cooperative system for transmission over time-selective channels. For example, given a target BER of $10^{-3}$, a performance gain of about $10 \mathrm{~dB}$ can be attained by the proposed MSDSD for a two-user cooperative system in a channel having a normalized Doppler frequency of 0.03 .
\end{abstract}

\section{INTRODUCTION}

Multiple antenna aided diversity techniques [1] constitute powerful techniques of mitigating the deleterious effects of fading, hence improving the end-to-end system performance, which is usually achieved by multiple co-located antenna elements at the transmitter and/or receiver. However, it is often impractical for the mobile to employ a large number of antennas for the sake of achieving a diversity gain due to its limited size. Furthermore, owing to the limited separation of the antenna elements, they rarely experience independent fading, which limits the achievable diversity gain and may be further compromised by the detrimental effects of the shadow fading, imposing further signal correlation amongst the antennas in each other's vicinity. Fortunately, in multi-user wireless systems cooperating mobiles may share their antennas in order to achieve uplink transmit diversity by formig a virtual antenna array (VAA) in a distributed fashion. Thus, so-called cooperative diversity relying on the cooperation among multiple terminals may be achieved [2].

On the other hand, in order to carry out classic coherent detection, channel estimation is required at the receiver, which relies on using training pilot signals and exploits the fact that in general, the consecutive channel impulse response (CIR) taps are correlated in time as governed by the vehicular speed, i.e. the Doppler frequency. However, channel estimation for an $M$-transmitter, $N$-receiver MIMO system requires the estimation of $(M \times N)$ CIRs, which may impose both an excessive complexity and a high pilot overhead, especially in mobile environments associated with relatively rapidly fluctuating channel conditions. Therefore, in such situations, differntially encoded transmissions combined with non-coherent detection requiring no channel state information (CSI) at the receiver becomes an attractive design alternative, leading to differential modulation assisted cooperative communications [3].

It is widely recognized at low mobile velocities that DAF-aided cooperative transmission [3] is capable of outperforming classic direct transmission using coherent detection. However, the performance of the former scheme rapidly degrades, as the channel connecting the multiple terminals becomes more time-selective and/or dispersive. Recently, in order to mitigate the error floor encountered by differentially encoded direct transmission combined with CDD employing an observation window size of $N_{\text {wind }}=2$, a multiplesymbol based differential sphere detection (MSDSD) technique using

Acknowledgements: The work reported in this paper has formed part of the Core 4 Research Programme of the Virtual Centre of Excellence in Mobile and Personal Communications, Mobile VCE, www.mobilevce.com, whose funding support, including that of EPSRC, is gratefully acknowledged.
$N_{\text {wind }}>2$ has been proposed in $[4,5]$. In the light of the above observations, our main contribution in this paper is the design of a MSDSD scheme proposed for multipath channels using multidimensional tree search [6], which is capable of making DAFaided cooperative systems significantly more robust to time-selective propagation environments.

\section{SYSTEM MOdEL}

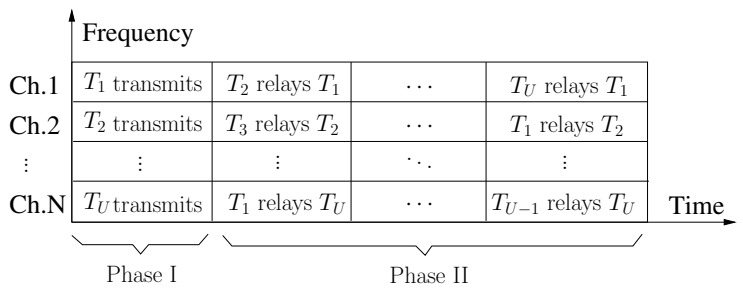

Fig. 1. Repetion-based channel allocation scheme.

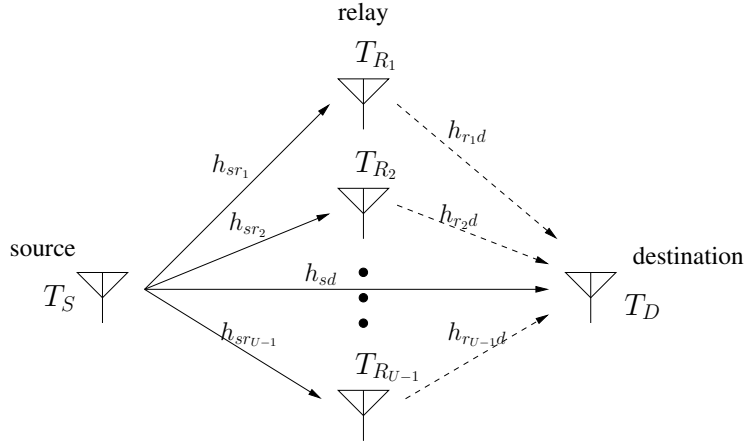

Fig. 2. Multiple-Relay-Node-Aided Cooperative Communication Scheme.

As depicted in Figure 1, we consider a $U$-user cooperationaided system, where signal transmission involves two transmission phases, namely, the broadcast phase-I and the relay phase-II. A user who directly sends his/her own information to the destination is regarded as a source node, while the other users who help forward the information received from the source node are considered as relay nodes. In both phases, any of the well-established multiple access scheme can be employed by the users to guarantee orthogonal transmission among them, such as for example time division multiple access (TDMA), frequency division multiple access (FDMA), or code division multiple access (CDMA). In this paper, TDMA is considered for the sake of simplicity. Furthermore, due to the symmetry of channel allocation among users, as indicated in Figure 1, we focus our attention on the information transmission of the source terminal $T_{S}$ seen in Figure 2, which potentially employs $(U-1)$ relay terminals $T_{R_{1}}, T_{R_{2}}, \cdots, T_{R_{U-1}}$ in order to achieve cooperative diversity by forming a VAA. Without loss of generality, we simply assume the employment of a single antenna for each terminal, and a unity total power $P$ shared by the collaborating mobiles for transmitting a symbol.

In order to avoid channel estimation in the user-cooperation-aided systems considered, the source node differentially encodes its information symbols $v_{s d}[n] \in \mathcal{M}_{c}=\left\{e^{j 2 \pi m / M} ; m=0,1, \cdots, M-1\right\}$, each of which contains $\log _{2} M$-bit information, as:

$$
\begin{aligned}
s_{s d}[n] & =\sqrt{P_{s}} \cdot s_{s d}[n-1] v_{s d}[n], \\
& =\sqrt{P_{s}} \cdot e^{j 2 \pi m / M}, m=0,1, \cdots, M-1,
\end{aligned}
$$


where $P_{s}$ is the source' transmit power. Thus, by having an overall transmit power constraint of unity, the total power required by all the $(U-1)$ relay nodes for forwarding the signal received from the source can be represented as $\sum_{u=1}^{U-1} P_{r_{u}}=1-P_{s}$, where $P_{r_{u}}$ is the power consumed by the $u$ th relay node. For the sake of mitigating the impairments imposed by the time-selective channels on the differential transmission, frame-based rather than symbol-based user-cooperation is carried out, which is achieved at the expense of both a higher detection delay and increased memory requirements. Hence, the source continuously broadcasts $L_{f}$ differentially encoded DPSK signals $s_{s d}^{l}[n],\left(n=0,1, \cdots, L_{f}-1\right)$ during phase-I, while the destination as well as the relay terminals receive and store them. In the ensuing phase-II, the DAF scheme [3] is employed by the relay node.

Without loss of generality, we now omitted the frame index $l$ in order to construct a single-symbol system model for the souce node's $n$th transmitted symbol in the context of the TDMA-based user-cooperation aided system of Figure 2 as:

$$
\mathbf{Y}_{n}=\mathbf{S}_{n} \mathbf{H}_{n}+\mathbf{W}_{n},
$$

where

$\mathbf{S}_{n}=\operatorname{diag}\left\{s_{s d}[n], s_{r_{1} d}\left[n+L_{f}\right], \cdots, s_{r_{U-1} d}\left[n+(U-1) L_{f}\right\}\right.$

represents the transmitted user-cooperation based signal matrix with the rows and columns denoting the spatial and temporal domains, respectively. The $\operatorname{diag}\{\cdot\}$ is the diagonalization operator. For the sake of simplicity, we consider a single-receive-antenna-aided destination node, which can be readily extended to a multiple-receive-antenna aided scenario. Hence, we have single-column matrices

$$
\begin{aligned}
\mathbf{Y}_{n} & =\left[\begin{array}{llll}
y_{s d}[n] & y_{r_{1} d}\left[n+L_{f}\right] & \cdots & y_{r_{U-1} d}\left[n+(U-1) L_{f}\right.
\end{array}\right]^{T}, \\
\mathbf{H}_{n} & =\left[\begin{array}{llll}
h_{s d}[n] & h_{r_{1} d}\left[n+L_{f}\right] & \cdots & h_{r_{U-1} d}\left[n+(U-1) L_{f}\right.
\end{array}\right]^{T},
\end{aligned}
$$

and

$$
\mathbf{W}_{n}=\left[w_{s d}[n] w_{r_{1} d}\left[n+L_{f}\right] \cdots w_{r_{U-1} d}\left[n+(U-1) L_{f}\right]\right]^{T},
$$

which correspond to the received signal matrix at the destination, to the channel matrix and to the AWGN matrix, respectively.

Additionally, the amplifed signal transmitted by the $u$ th relay node seen in Eq.(4) can be expressed as:

$$
\begin{aligned}
s_{r_{u} d}\left[n+r_{u} L_{f}\right] & =f_{A M_{r_{u}}} y_{s r_{u}}[n], \\
& =f_{A M_{r_{u}}}\left(s_{s d}[n] h_{s r_{u}}[n]+w_{s r_{u}}[n]\right),
\end{aligned}
$$

where $y_{s r_{u}}[n]$ is the received signal at the $u$ th relay node and $f_{A M_{r_{u}}}$ is the signal gain employed by the $u$ th relay node in order to ensure that the average transmit power of the $u$ th relay node becomes $P_{r_{u}}$, which is given by [3]:

$$
f_{A M_{r_{u}}}=\sqrt{\frac{P_{r_{u}}}{P_{s} \sigma_{s r_{u}}^{2}+N_{0}}},
$$

where $\sigma_{s r_{u}}^{2}$ is the variance of the channel's envelope between the source and the $u$ th relay node, which can be obtained by the longterm averaging of the received signals, and $N_{0}$ is the noise variance. Hence, by substituting Eq.(2) as well as Eq.(9) into Eq.(3), and following a number of straightforward manipulations, we arrive at the equivalent single-symbol system model expressed as:

$$
\mathbf{Y}_{n}=\tilde{\mathbf{S}}_{n} \tilde{\mathbf{H}}_{n}+\tilde{\mathbf{W}}_{n},
$$

where $\tilde{\mathbf{S}}_{n}, \tilde{\mathbf{H}}_{n}$ and $\tilde{\mathbf{W}}_{n}$, respectively, are given by Eq.(12), Eq.(13) and Eq.(14) at the top of the next page, which are the resultant equivalent transmitted user-cooperation signal matrix, the equivalent channel matrix and the equivalent noise matrix, respectively.

Here we define two diagonal matrices for storing the transmitpower-related factors of the DAF-aided system as below:

$$
\begin{aligned}
\mathbf{P}_{d} & =\operatorname{diag}\left\{\sqrt{P_{s}}, \sqrt{P_{r_{1}}}, \cdots, \sqrt{P_{r_{U-1}}}\right\}, \\
\mathbf{F}_{d A M} & =\operatorname{diag}\left\{1, \frac{f_{A M_{r_{1}}}}{\sqrt{P_{r_{1}}}}, \cdots, \frac{f_{A M_{r_{U-1}}}}{\sqrt{P_{r_{U-1}}}}\right\} .
\end{aligned}
$$

Furthermore, based on Eq.(12), (13) and (14), we can also construct the equivalent multiple-symbol system model as:

$$
\underline{\mathbf{Y}}=\underline{\tilde{\mathbf{S}}_{d}} \underline{\tilde{\mathbf{H}}}+\underline{\tilde{\mathbf{W}}},
$$

where the received signal block matrix $\underline{\mathbf{Y}}$, which contains $N_{\text {wind }}$ user-cooperation based received symbols corresponding to $N_{\text {wind }}$ consecutively transmitted differentially encoded symbols $s_{s d}[n],\left(n=0,1, \cdots, N_{\text {wind }}-1\right)$ by the source node, namely:

$$
\underline{\mathbf{Y}}=\left[\begin{array}{llll}
\mathbf{Y}_{n}^{T} & \mathbf{Y}_{n+1}^{T} & \cdots & \mathbf{Y}_{n+N_{\text {wind }}-1}^{T}
\end{array}\right]^{T},
$$

and the channel's block matrix $\tilde{\mathbf{H}}$ as well as the AWGN block matrix $\tilde{\tilde{\mathbf{W}}}$ are defined likewise by vertically concatenating $N_{\text {wind }}$ matrices $\tilde{\mathbf{H}}_{n},\left(n=0,1, \cdots, N_{\text {wind }}-1\right)$ and $\tilde{\mathbf{W}}_{n},\left(n=0,1, \cdots, N_{\text {wind }}-1\right)$, respectively. Therefore, we can represent $\underline{\tilde{\mathbf{H}}}$ as:

$$
\underline{\tilde{\mathbf{H}}}=\left[\begin{array}{llll}
\tilde{\mathbf{H}}_{n}^{T} & \tilde{\mathbf{H}}_{n+1}^{T} & \cdots & \tilde{\mathbf{H}}_{n+N_{\text {wind }}-1}^{T}
\end{array}\right]^{T},
$$

and express $\underline{\tilde{\mathbf{W}}}$ as:

$$
\underline{\tilde{\mathbf{W}}}=\left[\begin{array}{llll}
\tilde{\mathbf{W}}_{n}^{T} & \tilde{\mathbf{W}}_{n+1}^{T} & \cdots & \tilde{\mathbf{W}}_{n+N_{\text {wind }}-1}^{T}
\end{array}\right]^{T} .
$$

Moreover, the diagonal block matrix of the transmitted signal is constructed as:

$$
\underline{\tilde{\mathbf{S}}_{d}}=\operatorname{diag}\left\{\tilde{\mathbf{S}}_{n}, \tilde{\mathbf{S}}_{n+1}, \cdots, \tilde{\mathbf{S}}_{n+N_{\text {wind }}-1}\right\} .
$$

\section{Mutiple-Symbol Differential Sphere Detection} DESIGN FOR DAF-AIDED COOPERATIVE TRANSMISSIONS

\section{A. Principle of Multiple-Symbol Differential Detection}

Under the assumption that both the fading and noise are zero-mean complex Gaussian processes, the a priori probability function of $\underline{\mathbf{Y}}$ given by Eq.(18) can be expressed as:

$$
\operatorname{Pr}\left(\underline{\mathbf{Y}} \mid \underline{\tilde{\mathbf{S}}_{d}}\right)=\frac{\exp \left(-\operatorname{Tr}\left\{\underline{\mathbf{Y}}^{H} \underline{\Psi}^{-1} \underline{\mathbf{Y}}\right)\right.}{\left(\pi^{U N_{\text {wind }}} \operatorname{det}(\underline{\Psi})\right)},
$$

where the conditional autocorrelation matrix is given by:

$$
\begin{aligned}
\underline{\Psi} & =\mathcal{E}\left\{\underline{\mathbf{Y}}^{H} \mid \underline{\tilde{\mathbf{S}}}_{d}\right\}, \\
& =\underline{\tilde{\mathbf{S}}}_{d} \mathcal{E}\left\{\underline{\tilde{\mathbf{H}}}^{\tilde{\mathbf{H}}^{H}}\right\} \underline{\tilde{\mathbf{S}}}_{d}{ }^{H}+\mathcal{E}\left\{\underline{\tilde{\mathbf{W}} \tilde{\mathbf{W}}^{H}}\right\} .
\end{aligned}
$$

Note that we consider Rayleigh fading having an autocorrelation function of $\varphi^{t}[\kappa] \triangleq \mathcal{E}\left\{h[n+\kappa] h^{*}[n]\right\}=J_{0}\left(2 \pi f_{d} \kappa\right)$ according to the widely-used Clarke model, where $J_{0}(\cdot)$ and $f_{d}$ represent the zero-order Bessel function of first kind and the normalized Doppler frequency, respectively. Thus, in the context of the DAF-aided usercooperation system of Figure 2, the autocorrelation matrices of the channel and the noise are given by Eq.(25) and Eq.(27) at the top of the next page.

With the aid of Bayes' theorem [7], the decision metric of the MLMSDD designed for the DAF-aided user-cooperation aided system of Figure 2 can be expressed as:

$$
\underline{\hat{\mathbf{S}}_{M L}}=\arg \min _{\underline{\tilde{\mathbf{s}} \in \mathcal{C}^{N} \text { wind }}} \operatorname{Tr}\left\{\underline{\mathbf{Y}}^{H} \underline{\Psi}^{-1} \underline{\mathbf{Y}}\right\},
$$

where $\mathcal{C}$ is the user-cooperation based signal constellation set.

\section{B. Multiple-Symbol Differential Sphere Detection Design}

1) Transformation of the ML-MSDD Metric: The equivalent usercooperation based transmitted signal matrix $\tilde{\mathbf{S}}_{d}$ as constructed in Eq.(12) for the DAF-aided cooperative system is a unitary matrix, hence we have:

$$
\underline{\tilde{\mathbf{S}}}_{d}{ }^{-1}={\underline{\tilde{\mathbf{S}}_{d}}}^{H} .
$$

Additionally, since the noise contributions imposed at the relay and destination nodes are both temporally and spatially uncorrelated, thus the autocorrelation $\mathcal{E}\left\{\underline{\tilde{\mathbf{W}}} \tilde{\mathbf{W}}^{H}\right\}$ is a diagonal matrix. Hence we can reformulate Eq.(24) as:

$$
\begin{aligned}
\underline{\Psi} & =\underline{\tilde{\mathbf{S}}_{d}}\left(\mathcal{E}\left\{\underline{\tilde{\mathbf{H}}}^{\tilde{\mathbf{H}}^{H}}\right\}+\mathcal{E}\left\{\underline{\left.\left.\tilde{\mathbf{W}} \tilde{\mathbf{W}}^{H}\right\}\right) \underline{\tilde{\mathbf{S}}}_{d}{ }^{H},}\right.\right. \\
& =\underline{\tilde{\mathbf{S}}_{d}} \mathbf{C} \underline{\tilde{\mathbf{S}}}_{d}{ }^{H},
\end{aligned}
$$




$$
\tilde{\mathbf{S}}_{n}=\left[\begin{array}{cccc}
e^{j 2 \pi m / M} & 0 & \cdots & 0 \\
0 & e^{j 2 \pi m / M} & \cdots & 0 \\
\vdots & \vdots & \ddots & \vdots \\
0 & 0 & \cdots & e^{j 2 \pi m / M}
\end{array}\right]_{U \times U}
$$

where $m=0,1, \cdots, M-1$.

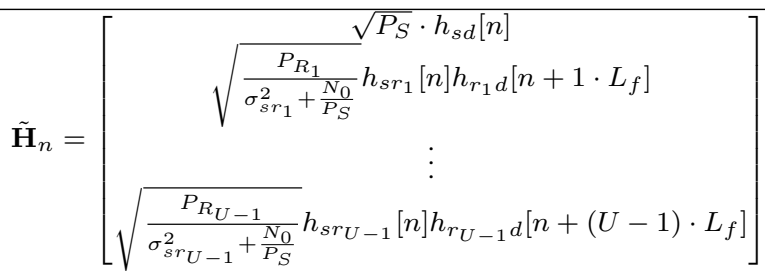

where

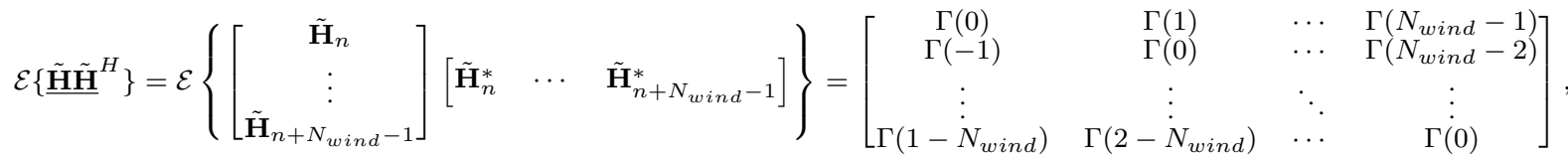

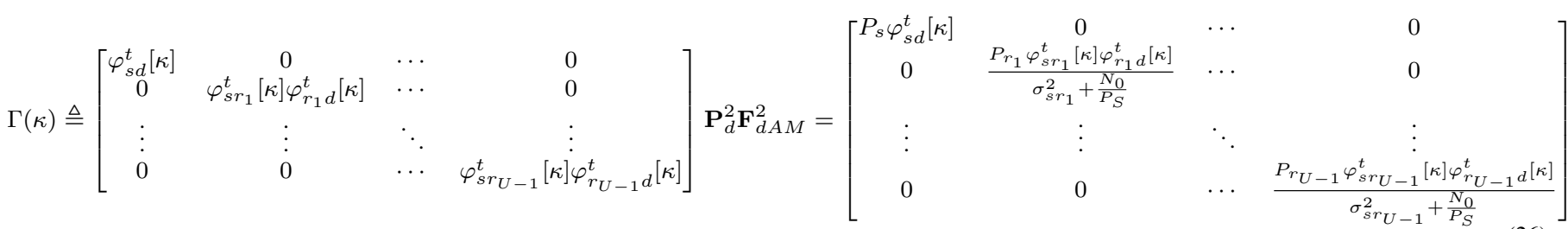

$$
\mathcal{E}\left\{\underline{\tilde{\mathbf{W}}} \tilde{\tilde{\mathbf{W}}}^{H}\right\}=\mathbf{I}_{N_{w i n d}} \otimes\left[\begin{array}{cccc}
N_{0} & 0 & \cdots & 0 \\
0 & \left(\frac{P_{R_{1}} \sigma_{r_{1} d}^{2}}{P_{S} \sigma_{s r_{1}}^{2}+N_{0}}+1\right) N_{0} & \cdots & 0 \\
\vdots & \vdots & \ddots & \vdots \\
0 & 0 & \cdots & \left(\frac{P_{R_{U-1}} \sigma_{r_{U-1} d}^{2}}{P_{S} \sigma_{s r_{U-1}}^{2}+N_{0}}+1\right) N_{0}
\end{array}\right]
$$

where we have

$$
\mathbf{C} \triangleq \mathcal{E}\left\{\underline{\tilde{\mathbf{H}}}^{\tilde{\mathbf{H}}^{H}}\right\}+\mathcal{E}\left\{\underline{\tilde{\mathbf{W}}}_{\tilde{\mathbf{W}}}^{H}\right\},
$$

which is defined as the $\left(U N_{\text {wind }} \times U N_{\text {wind }}\right)$-element channel-noise autocorrelation matrix. Then, the metric of the ML-MSDD can be reformulated by substituting Eq.(31) characterizing $\Psi$ in Eq.(28) and using Eq.(29), thus we arrive at:

$$
\begin{aligned}
\underline{\hat{\mathbf{S}}_{M L}} & =\arg \min _{\underline{\tilde{\mathbf{s}} \in \mathcal{C}^{N} \text { wind }}} \operatorname{Tr}\left\{\underline{\mathbf{Y}}^{H}\left(\underline{\tilde{\mathbf{S}}_{d}} \underline{\mathbf{C}}_{d}{ }^{H}\right)^{-1} \underline{\mathbf{Y}}\right\}, \\
& =\arg \min _{\underline{\tilde{\mathbf{s}} \in \mathcal{C}^{N} \text { wind }}} \operatorname{Tr}\left\{\underline{\mathbf{Y}^{H}} \underline{\tilde{\mathbf{S}}}_{d} \mathbf{C}^{-1} \underline{\tilde{\mathbf{S}}_{d}}{ }^{H} \underline{\mathbf{Y}}\right\} .
\end{aligned}
$$

Let us now introduce two operators. First, a matrix-to-vector transformation operator $T_{c}(\cdot)$ is defined for an arbitary square matrix

$$
\mathbf{A}_{n}=\left[\begin{array}{cccc}
a_{n, 1} & a_{n, U+1} & \cdots & a_{n,(U-1) U+1} \\
a_{n, 2} & a_{n, U+2} & \cdots & a_{n,(U-1) U+2} \\
\vdots & \vdots & \ddots & \vdots \\
a_{n, U} & a_{n, 2 U} & \cdots & a_{n, U^{2}}
\end{array}\right]_{U \times U}
$$

as:

$$
T_{c}\left(\mathbf{A}_{n}\right)=\left[\begin{array}{lllll}
a_{n, 1} & a_{n, 2} & \cdots & a_{n, U^{2}-1} & a_{n, U^{2}}
\end{array}\right]^{T} .
$$

When $T_{c}(\cdot)$ is applied to a diagonal block matrix of the form

$$
\underline{\mathbf{A}_{d}}=\operatorname{diag}\left\{\mathbf{A}_{0}, \mathbf{A}_{1}, \cdots, \mathbf{A}_{N_{w i n d}-1}\right\},
$$

we have

$$
T_{c}\left(\underline{\mathbf{A}_{d}}\right)=\left[T_{c}\left(\mathbf{A}_{0}\right)^{T} T_{c}\left(\mathbf{A}_{1}\right)^{T} \cdots T_{c}\left(\mathbf{A}_{N_{\text {wind }}-1}\right)^{T}\right]^{T},
$$

which converts the diagonal block matrix to a column vector by ignoring its null elementary block matrices having values of zero.
Next, we introduce another transformation operator $T_{d}(\cdot)$ for a single-column matrix $\underline{\mathbf{B}}$ constituted by $N_{\text {wind }}$ number of $(U \times 1)$ element sub-matrices $\overline{\mathbf{B}}_{n},\left(n=0,1, \cdots, N_{\text {wind }}-1\right)$, which can be expressed as:

$$
\underline{\mathbf{B}}=\left[\begin{array}{llll}
\mathbf{B}_{0}^{T} & \mathbf{B}_{1}^{T} & \cdots & \mathbf{B}_{N_{\text {wind }}-1}^{T}
\end{array}\right]^{T} .
$$

When the transformation operation $T_{d}(\cdot)$ is applied to $\underline{\mathbf{B}}$, we arrive at:

$$
T_{d}(\underline{\mathbf{B}})=\left[\begin{array}{cccc}
\boldsymbol{\Upsilon}_{0} & 0 & \cdots & 0 \\
0 & \boldsymbol{\Upsilon}_{1} & \cdots & 0 \\
\vdots & \vdots & \ddots & \vdots \\
0 & 0 & \cdots & \boldsymbol{\Upsilon}_{N_{\text {wind }}-1}
\end{array}\right]
$$

where

$$
\mathbf{\Upsilon}_{n} \triangleq\left[\begin{array}{cccc}
\mathbf{B}_{n}^{T} & 0 & \cdots & 0 \\
0 & \mathbf{B}_{n}^{T} & \cdots & 0 \\
\vdots & \vdots & \ddots & \vdots \\
0 & 0 & \cdots & \mathbf{B}_{n}^{T}
\end{array}\right]
$$

which has $\left(U \times U^{2}\right)$ elements. Consequently, the resultant matrix $T_{d}(\underline{\mathbf{B}})$ constains $N_{\text {wind }}$ block matrices $\boldsymbol{\Upsilon}_{n}\left(n=1,2, \cdots, N_{\text {wind }}\right)$ along the main diagonal of Eq.(40), leading to $\left(U N_{\text {wind }} \times U^{2} N_{\text {wind }}\right)$ elements.

Consequently, given the transformation operators $T_{c}(\cdot)$ defined in Eq.(36) and Eq.(37) as well as $T_{d}(\cdot)$ defined in Eq.(40), we can 
reformulate the ML-MSDD metric of Eq.(34) as:

$$
\begin{aligned}
& \underline{\hat{\mathbf{S}}_{M L}} \\
= & \arg \min _{\underline{\tilde{\mathbf{s}}} \in \mathcal{C}^{N_{\text {wind }}}} \operatorname{Tr}\left\{\underline{\mathbf{Y}}{ }^{H} \underline{\tilde{\mathbf{S}}_{d}} \mathbf{C}^{-1} \underline{\tilde{\mathbf{S}}}_{d}^{H} \underline{\mathbf{Y}}\right\}, \\
= & \arg \min _{\underline{\tilde{\mathbf{s}}} \in \mathcal{C}^{N_{\text {wind }}}} \operatorname{Tr}\left\{\left(T_{d}(\underline{\mathbf{Y}}) T_{c}\left(\underline{\tilde{\mathbf{S}}_{d}}{ }^{*}\right)\right)^{H} \mathbf{C}^{-1} T_{d}(\underline{\mathbf{Y}}) T_{c}\left(\underline{\tilde{\mathbf{S}}_{d}}{ }^{*}\right)\right\}, \\
= & \arg \min _{\underline{\tilde{\mathbf{s}}} \in \mathcal{C}^{N_{\text {wind }}}} \operatorname{Tr}\left\{T_{c}\left(\underline{\tilde{\mathbf{S}}_{d}}\right) T_{d}(\underline{\mathbf{Y}})^{H} \mathbf{C}^{-1} T_{d}(\underline{\mathbf{Y}}) T_{c}\left(\underline{\tilde{\mathbf{S}}}_{d}^{*}\right)\right\} .
\end{aligned}
$$

2) Channel-Noise Autocorrelation Matrix Triangularization: Let us now generate the $\left(U N_{\text {wind }} \times U N_{\text {wind }}\right)$-element upper-triangular matrix $\mathbf{F}$, which satisfies $\mathbf{F}^{H} \mathbf{F}=\mathbf{C}^{-1}$ with the aid of Cholesky factorization. Then we arrive at:

$$
\underline{\hat{\mathbf{S}}_{M L}}=\arg \min _{\underline{\tilde{\mathbf{s}} \in \mathcal{C}^{N} \text { wind }}} \operatorname{Tr}\left\{T_{c}\left(\underline{\tilde{\mathbf{S}}_{d}}\right) T_{d}(\underline{\mathbf{Y}})^{H} \mathbf{F}^{H} \mathbf{F} T_{d}(\underline{\mathbf{Y}}) T_{c}\left(\underline{\tilde{\mathbf{S}}_{d}}{ }^{*}\right)\right\},
$$

Then, by further defining a $\left(U N_{\text {wind }} \times U^{2} N_{\text {wind }}\right)$-element matrix $\mathrm{U}$ as:

$$
\begin{aligned}
\mathbf{U} & \triangleq\left(\mathbf{F} T_{d}(\underline{\mathbf{Y}})\right)^{*}, \\
& =\left[\begin{array}{cccc}
\mathbf{U}_{1,1} & \mathbf{U}_{1,2} & \ldots & \mathbf{U}_{1, N_{\text {wind }}} \\
\mathbf{0} & \mathbf{U}_{2,2} & \cdots & \mathbf{U}_{2, N_{\text {wind }}} \\
\vdots & \vdots & \ddots & \vdots \\
\mathbf{0} & \mathbf{0} & \cdots & \mathbf{U}_{N_{\text {wind }}, N_{\text {wind }}}
\end{array}\right],
\end{aligned}
$$

where

$$
\begin{aligned}
& \mathbf{U}_{n, n} \triangleq \\
& {\left[\begin{array}{cccc}
u_{U(n-1)+1, U^{2}(n-1)+1} & u_{U(n-1)+1, U^{2}(n-1)+2} & \cdots & u_{U(n-1)+1, U^{2} n} \\
u_{U(n-1)+2, U^{2}(n-1)+1} & u_{U(n-1)+2, U^{2}(n-1)+2} & \cdots & u_{U(n-1)+2, U^{2} n} \\
\vdots & \vdots & \ddots & \vdots \\
u_{U n, U^{2}(n-1)+1} & u_{U n, U^{2}(n-1)+2} & \cdots & u_{U n, U^{2} n}
\end{array}\right]}
\end{aligned}
$$

we finally arrive at:

$$
\begin{aligned}
& \underline{\hat{\mathbf{S}}_{M L}}=\arg \min _{\underline{\tilde{\mathbf{s}} \in \mathcal{C}^{N} w_{\text {wind }}}}\left\|\mathbf{U} T_{c}\left(\underline{\tilde{\mathbf{S}}_{d}}\right)\right\|^{2}, \\
& =\arg \min _{\tilde{\mathbf{s}} \in \mathcal{M}_{c}^{\left(U^{2} N_{w i n d}\right)}}\|\mathbf{U} \tilde{\mathbf{s}}\|^{2},
\end{aligned}
$$

where

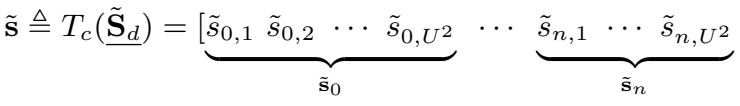

$$
\begin{aligned}
& \cdots \underbrace{\tilde{s}_{N_{\text {wind }}-1,1} \cdots \tilde{s}_{N_{\text {wind }}-1, U^{2}}}_{\tilde{\mathbf{s}}_{N_{\text {wind }}-1}}]^{T},
\end{aligned}
$$

which completes the process of transforming the ML-MSDD metric of Eq.(28) to a shortest-vector problem.

3) Multi-Dimensional Tree Search in a Confined-Radius HyperSphere: In order to search for the ML solution of Eq.(51) in a confined hypersphere at an affordable computational complexity, an initial search radius $C$ is introduced. Thus, we obtain the searchmetric of the MSDSD as:

$$
\begin{aligned}
& \frac{\hat{\mathbf{S}}_{M S D S D}}{\arg \min _{\tilde{\mathbf{s}} \in \mathcal{M}_{c}^{\left(U^{2} N_{\text {wind }}\right)}}}\|\mathbf{U} \tilde{\mathbf{s}}\|^{2} \leq C^{2}, \\
= & \arg \min _{\tilde{\mathbf{s}} \in \mathcal{M}_{c}^{\left(U^{2} N_{\text {wind }}\right)}}\left\|\sum_{n=1}^{N_{\text {wind }}}\left(\sum_{m=n}^{N_{\text {wind }}} \mathbf{U}_{n, m} \tilde{\mathbf{s}}_{m}\right)\right\|^{2} \leq C^{2} .
\end{aligned}
$$

Without loss of generality we now simply consider a TDMA-aided two-user cooperation assisted system $(U=2)$, where each of the $N_{\text {wind }}$ matrices $\tilde{\mathbf{S}}_{n}\left(n=1,2, \cdots, N_{\text {wind }}\right)$ is a diagonal one, as seen in Eq.(12), where we have:

$$
\tilde{s}_{n, 2}=\tilde{s}_{n, 3}=0 .
$$

Thus, the sub-vector $\tilde{\mathbf{s}}_{n}$ in Eq.(52) can be expressed as:

$$
\tilde{\mathbf{s}}_{n}=\left[\begin{array}{llll}
\tilde{s}_{n, 1} & 0 & 0 & \tilde{s}_{n, 4}
\end{array}\right] .
$$

Moreover, in the scenario of the two-user cooperation-aided system, the sub-block matrix $U_{n, n}$ of Eq.(49) becomes as:

$$
\begin{aligned}
\mathbf{U}_{n, n} & =\left[\begin{array}{cccc}
u_{2 n-1,4 n-3} & u_{2 n-1,4 n-2} & u_{2 n-1,4 n-1} & u_{2 n-1,4 n} \\
u_{2 n, 4 n-3} & u_{2 n, 4 n-2} & u_{2 n, 4 n-1} & u_{2 n, 4 n}
\end{array}\right], \\
& =\left[\begin{array}{cccc}
u_{2 n-1,4 n-3} & u_{2 n-1,4 n-2} & 0 & 0 \\
0 & 0 & u_{2 n, 4 n-1} & u_{2 n, 4 n}
\end{array}\right],
\end{aligned}
$$

due to the fact that the upper-triangular matrix $\mathbf{F}$ is multiplied by the matrix $T_{d}(\mathbf{Y})$, which has the specific matrix structure seen in Eq.(40).

In the sequel, with the aid of Eq.(56) and Eq.(58), Eq.(54) can be further streamlined by omitting the terms associated with the nulls in the sub-vector $\tilde{\mathbf{s}}_{n}$ and the sub-block matrix $\mathbf{U}_{n, n}$, yielding:

$$
\begin{aligned}
\underline{\hat{\mathbf{S}}_{M L}} & =\arg \min _{\tilde{s}_{n, 1}, \tilde{s}_{n, 4} \in \mathcal{M}_{c}} \sum_{n=1}^{N_{\text {wind }}} \mid \underbrace{\mid u_{2 n-1,4 n-3} \tilde{s}_{n, 1}+\sum_{m=n+1}^{N_{\text {wind }}} u_{2 n-1,4 m-3} \tilde{s}_{m, 1}}_{1^{\text {st }} \text { Layer }} \\
& +\left.\underbrace{u_{2 n, 4 n} \tilde{s}_{n, 4}+\sum_{m=n+1}^{N_{\text {wind }}} u_{2 n, 4 m} \tilde{s}_{m, 4}}_{2^{\text {nd }} \text { Layer }}\right|^{2} \leq C .
\end{aligned}
$$

Furthermore, since the DAF scheme is employed, $\tilde{s}_{n, 1}$ and $\tilde{s}_{n, 4}$ not only share an identical legitimate candidate pool $\mathcal{C}=\left\{e^{j 2 \pi i / M}, i=\right.$ $0,1, \cdots, M-1\}$, but they also have the same trial value each time during the tree search, namely, we have:

$$
\tilde{s}_{n, 1}=\tilde{s}_{n, 4}=e^{j 2 \pi i / M}, i=0,1, \cdots, M-1 .
$$

Hence we can further simplify Eq.(59) as:

$$
\begin{aligned}
\underline{\hat{\mathbf{S}}_{M L}} & =\arg \min _{\tilde{s}_{n, 1} \in \mathcal{M}_{c}} \sum_{n=1}^{N_{\text {wind }}} \mid\left(u_{2 n-1,4 n-3}+u_{2 n, 4 n}\right) \tilde{s}_{n, 1} \\
& +\left.\sum_{m=n+1}^{N_{\text {wind }}}\left(u_{2 n-1,4 m-3}+u_{2 n, 4 m}\right) \tilde{s}_{m, 1}\right|^{2} \leq C .
\end{aligned}
$$

Hence, the original two-layer tree search carried out by the MSDSD is effectively reduced to the conventional single-layer tree search from $n=N_{\text {wind }}$ to $n=1$, which may be implemented by the conventional sphere detection (SD) algorithm of [8,9], in the DAF-aided two-user cooperation scenario.

\section{Simulation Results and Discussion}

It is well-understood that the DPSK-aided non-cooperative system employing the $\mathrm{CDD}$ suffers from a $3 \mathrm{~dB}$ performance loss in comparison to its coherent-modulation-assisted counterpart in the context of slow fading channels, i.e. when experiencing a normalized Doppler frequency of $f_{d}=0.001$, as seen in Figure 3. Thanks to the DAF scheme, the two-user cooperation-aided system, which attains the maximum achievable diversity order of two, is capable of outperforming both of the above-mentioned non-cooperative systems without requiring high-complexity channel estimation. However, the time-selectivity of the fast-fading channel may severely impair the achievable performance of the CDD at the destination node. More explicitly, observe in Figure 3 that the two-user cooperation-aided system encountered an error floor for $f_{d}=0.03$. To be more specific, the corresponding BER curve levels out just below $10^{-3}$, as $P / N_{0}$ increases. For the sake of exploiting the correlation between the channel-induced phase-rotation experienced by multiple consecutive transmitted DPSK symbols and hence to reduce the detrimental impact imposed by the time-selective channel on the DAF-aided system considered, the proposed MSDSD using $N_{\text {wind }}=11$ was employed by the destination node at the expense of a higher 


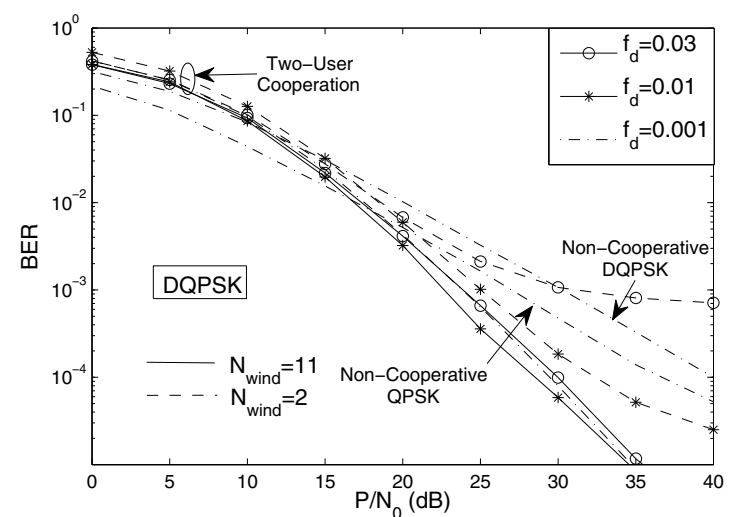

Fig. 3. BER performance improvement achieved by the MSDSD employing $N_{\text {wind }}=11$ for the DAF-aided T-DQPSK-modulated two-user cooperative system in a time-selective Rayleigh fading channel.

computational complexity. Remarkably, the error floor encountered by the system employing the CDD in fast-fading channels was essentially eliminated. For example, the BER curve corresponding to the MSDSD-aided cooperative system obtained for $f_{d}=0.03$ coincides with that of its CDD-aided counterpart, which was recorded for a relatively slowly-fading channel associated with $f_{d}=0.001$, resulting in a performance gain of more than $10 \mathrm{~dB}$, at a target BER of $10^{-3}$. Furthermore, the former system operating in a fast fading channel having $f_{d}=0.01$ is capable of outperforming the latter, even if the latter is operating in a slow fading channel having $f_{d}=0.001$. Therefore, even in the presence of a severely time-selective channel, the DAF-aided user-cooperation associated system employing the MSDSD is capable of achieving a desirable performance by jointly differentially detecting a sufficiently high number of consecutively received user-cooperation based symbols $\mathbf{Y}_{n}\left(n=0,1, \cdots, N_{\text {wind }}-1\right)$ as formulated in Eq.(3) with the knowledge of the channel-noise autocorrelation matrix $\mathbf{C}$ of Eq.(32), which characterizes the CIR statistics of both the direct and relay links together with the noise statistics.

\begin{tabular}{|c|c|c|c|}
\hline & $f_{d, s d}$ & $f_{d, s r}$ & $f_{d, r d}$ \\
\hline Scenario I & 0.03 & 0.03 & 0.001 \\
\hline Scenario II & 0.001 & 0.03 & 0.03 \\
\hline Scenario III & 0.03 & 0.001 & 0.03 \\
\hline
\end{tabular}

TABLE I

NORMALIZED DOPPLER FREQUENCY ALLOCATION OF THREE DIFFERENT SCENARIOS.

The previously described simulations were carried out under the assumption that an identical normalized Doppler frequency is exhibited by each link of the user-cooperation aided system, namely that we have $f_{d, s d}=f_{d, s r}=f_{d, r d}=f_{d}$. However, a more realistic scenario is the one where the relative speeds of all the cooperative users as well as of the destination terminal are different from each other, leading to a different Doppler frequency for each link. Thus, in order to investigate the impact of different relative speeds among all the nodes on the attainable performance of the DAF-aided system, Monte Carlo simulations were carried out for the three different scenarios summarized in Table I. In all the three situations, only one of the three nodes in the two-user cooperation-aided system is supposed to move relative to the other two nodes at a speed resulting in a normalized Doppler frequency of 0.03 , while the latter two remain immobile relative to each other yielding, a normalized Doppler frequency of 0.001 .

In Figure 4 the BER curves corresponding to the three different scenarios of Table I are bounded by the two dashed-dotted BER curves having no legends, which were obtained by assuming an identical normalized Doppler frequency of $f_{d}=0.03$ and $f_{d}=0.001$ for each link in the user-cooperation aided system, respectively. This is not unexpected, since the two above-mentioned BER bounds correspond to the least and most desirable time-selective channel conditions considered in this paper, respectively. The channel quality

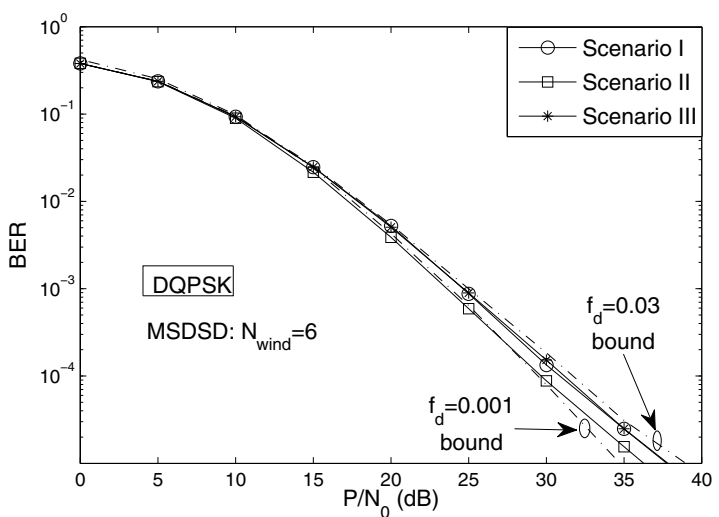

Fig. 4. The impact of the relative mobility among the source, relay and destination nodes on the BER performance of the DAF-aided T-DQPSKmodulated two-user cooperative system employing MSDSD at the destination node in Rayleigh fading channels.

of the direct link characterized in terms of its grade of time-selectivity predetermines the achievable performance of the DAF-aided usercooperation system employing the MSDSD. Hence, it is observed in Figure 4 that the system is capable of attaining a better BER performance in Scenario II $\left(f_{d, s d}=0.001\right)$ than in the other two scenarios $\left(f_{d, s d}=0.03\right)$. However, as seen in Figure 4 , due to the high speed of the relay node observed in Scenario II between the source and destination nodes, the MSDSD employing $N_{\text {wind }}=6$ remains unable to completely eliminate the impairments induced by the time-selective channel, unless a higher $N_{w i n d}$ value is employed. Therefore, a modest performance degradation occurs in comparison to the $f_{d}=0.001$ scenario. On the other hand, the MSDSD-aided system exhibits a similar performance in Scenario I and Scenario III, since the source-relay and relay-destination links are symmetric and thus they are exchangable in the context of the DAF scheme, as observed in Eq.(26).

\section{CONCLUSION}

A MSDSD scheme was proposed for mitigating the error floor encountered by the DAF-aided user-cooperation aided system in timeselective channels, leading to a significant performance gain over the system using the CDD. For example, given a target BER of $10^{-3}$, a performance gain of about $10 \mathrm{~dB}$ can be attained by the proposed MSDSD employing $N_{\text {cand }}=11$ for a DQPSK modulated cooperative two-user system in a relatively fast-fading channel having a normalized Doppler frequency of 0.03 .

\section{REFERENCES}

[1] G. J. Foschini and M. J. Gans, "On limits of wireless communications in a fading environment when using multiple antennas," Wireless Personal Communications, vol. 6, pp. 311-335, Mar. 1998

[2] J. N. Laneman, D. N. C. Tse, and G. W. Wornell, "Cooperative diversity in wireless networks: Efficient protocols and outage behavior," IEEE Transaction on Information Theory, vol. 50, pp. 3062-3080, Dec. 2004.

[3] T. Himsoon, W. Su, and K. J. R. Liu, "Differential transmission for amplify-and-forward cooperative communications," IEEE Signal Processing Letters, vol. 12, pp. 597-600, Sept. 2005.

[4] L. Lampe, R. Schober, V. Pauli, and C. Windpassinger, "Multiple-symbol differential sphere decoding," IEEE Transactions on Communications, vol. 12, pp. 1981-1985, Dec. 2005.

[5] V. Pauli and L. Lampe, "Multiple-symbol differential sphere decoding for unitary space-time modulation," IEEE Global Telecommunications Conference, vol. 3, p. 6, Nov. 2005.

[6] L. Wang, O. Alamri, and L. Hanzo, " $K$-best sphere detection for the sphere packing modulation aided SDMA/OFDM uplink," to appear in Proceedings of IEEE GLOBECOM'O8.

[7] L. Hanzo, M. Munster, B. J. Choi, and T. Keller, OFDM and MC-CDMA for Broadband Multi-User Communications, WLANs and Broadcasting. IEEE Press, 2003.

[8] M. O. Damen, H. E. Gamal, and G. Caire, "On maximum-likelihood detection and the search for the closest lattice point," IEEE Transactions on Information Theory, vol. 49, pp. 2389-2402, Oct. 2003.

[9] L. Wang, L. Xu, S. Chen, and L. Hanzo, "Three-stage irregular convolutional coded iterative center-shifting $k$-best sphere detection for softdecision SDMA-OFDM," accepted for publication in the IEEE Transactions on Vehicular Technology. 